\title{
IMPLICIT THREE-DIMENSIONAL GEO-MODELLING BASED ON HRBF SURFACE
}

\author{
Jiateng Gou ${ }^{\mathrm{a}}$, Wenhui Zhou ${ }^{\mathrm{a}}$, Lixin Wu ${ }^{\mathrm{a}, \mathrm{b}}$ \\ ${ }^{a}$ College of Resources and Civil Engineering, Northeastern University, Shenyang 110819, China - guojiateng@ mail.neu.edu.cn, \\ zhouwenhuimail@163.com \\ ${ }^{\mathrm{b}}$ School of Environmental Science and Spatial Information, China University of Mine \& Technology, Xuzhou 221008, China - \\ awulixin@263.net
}

KEY WORDS: Implicit modelling, Hermite radial basis function (HRBF), 3D geological modelling, ore body modelling

\begin{abstract}
:
Three-dimensional (3D) geological models are important representations of the results of regional geological surveys. However, the process of constructing 3D geological models from two-dimensional (2D) geological elements remains difficult and time-consuming. This paper proposes a method of migrating from 2D elements to 3D models. First, the geological interfaces were constructed using the Hermite Radial Basis Function (HRBF) to interpolate the boundaries and attitude data. Then, the subsurface geological bodies were extracted from the spatial map area using the Boolean method between the HRBF surface and the fundamental body. Finally, the top surfaces of the geological bodies were constructed by coupling the geological boundaries to digital elevation models. Based on this workflow, a prototype system was developed, and typical geological structures (e.g., folds, faults, and strata) were simulated. Geological modes were constructed through this workflow based on realistic regional geological survey data. For extended applications in 3D modelling of other kinds of geo-objects, mining ore body models and urban geotechnical engineering stratum models were constructed by this method from drill-hole data. The model construction process was rapid, and the resulting models accorded with the constraints of the original data.
\end{abstract}

\section{Introduction}

Three-dimensional (3D) modelling of geo-objects, such as subsurface stratum, deep geological structures and ore body, plays a very important role in the geological survey results expression, mine predication and exploitation, underground resources evaluation etc. Since the proposal of 3D geoscience modelling in 1990s (Houlding S., 1994), there has been a series of proposed geological modelling methods, including section connection (Ming, J., 2010), GTP method (Wu L., 2004) and free form surface method (De Kemp E A, 2003) etc. Based on these methods, a number of geological modelling systems were developed (Qingyuan Li, 2013), such as GOCAD (Zanchi A, 2009), GeoModeller (Vance T C, 2007), GSI3D (Kessler, H., 2009 ) etc. By now, these schemes have been widely applied in seismic hazard evaluation (Alaei B., 2012) geological statistical analysis (Raiber M, 2012), groundwater assessment (Borghi A, 2015), Geophysical inversion modelling (McGaughey J, 2007) and geothermal exploration (Calcagno P., 2015). However, most of existing methods require mass manual interactions in the modelling process unavoidably, of which the modelling efficient was relatively unsatisfactory (Kessler, H., 2009). Meanwhile, the models were very difficult to update when data changed, requiring repetitive and boring manual interaction (Cowan E J, 2003). As stated above, more convenient, faster and more precise modelling methods are desiderated to be promoted in $3 \mathrm{D}$ geological modelling.

With the development of surface technology based on coordinates and vectors interpolating, implicit function surface was introduced as a novel method into 3D geological modelling (Kazhdan M, 2006; Amorim, R., 2014). Implicit function ensured the positional conformity between reconstructed surface and the constrained points and vectors. Meanwhile, due to the three degrees of freedom in the 3D space treated as independent variables in the function, the surface could simulate complex spatial objects more flexibly and easily. Among these implicit surfaces, the radial basis surface is especially adaptable in 3D geological modelling, which took coordinates and vectors as constraint conditions to construct a spatial surface and was adopted in mining industry, 3D geological mapping etc.

\section{Methodology}

Because of the importance of vector data, such as geological attitudes, Hermite Radial Basis Function (HRBF, as formula 1) surface (Macedo, I., 2011) was preferred and adopted to complete 3D geological mapping, ore body simulation and subsurface stratum modelling in our study.

$$
f(x)=\sum_{i=1}^{n}\left(\alpha_{i} \varphi\left(x-x_{i}\right)-\left\langle\beta_{i}, \nabla \varphi\left(x-x_{i}\right)\right\rangle\right)
$$

where $\varphi(x)=\emptyset(\|x\|)$ is the radial basis function (in this paper, the global support radial basis function $\left.\emptyset(t)=t^{3}\right)$ and $\alpha_{i} \in R, \beta_{i} \in R^{3}$ are unknowns and can be uniquely determined by the coordinates and gradients of the boundary points. The equations used to calculate these unknowns are

$$
\left\{\begin{array}{l}
f\left(x_{j}\right)=\sum_{i=1}^{n}\left(\alpha_{i} \varphi\left(x_{j}-x_{i}\right)-\left\langle\beta_{i}, \nabla \varphi\left(x_{j}-x_{i}\right)\right\rangle\right)=0 \\
\nabla f\left(x_{j}\right)=\sum_{i=1}^{n}\left(\alpha_{i} \nabla \varphi\left(x_{j}-x_{i}\right)-H \varphi\left(x_{j}-x_{i}\right) \beta_{i}\right)=n_{j}
\end{array}\right.
$$

where $\mathrm{H}$ is the Hess operator:

$$
H=\left[\begin{array}{ccc}
\frac{\partial^{2}}{\partial x \partial x} & \frac{\partial^{2}}{\partial x \partial y} & \frac{\partial^{2}}{\partial x \partial z} \\
\frac{\partial^{2}}{\partial y \partial x} & \frac{\partial^{2}}{\partial y \partial y} & \frac{\partial^{2}}{\partial y \partial z} \\
\frac{\partial^{2}}{\partial z \partial x} & \frac{\partial^{2}}{\partial z \partial y} & \frac{\partial^{2}}{\partial z \partial z}
\end{array}\right]
$$

The equations can be transformed as

$$
A \lambda=c
$$

where $A$ is a $4 n \times 4 n$ matrix and $\lambda$ and $c$ are $4 n$ vectors. In this method, $n$ points provide $4 n$ equations and $4 n$ unknowns. Through the inverse matrix of A, $\lambda$ can be calculated as

$$
\lambda=A^{-1} c
$$

The HRBF implicit surface's parameters were then acquired, and the surface was uniquely determined.

Through HRBF surface, a novel 3D geological mapping 
method based on 2D geological map was proposed (Guo J., 2016). To maintain the topological relations between the geological regions in the 2D map, an extraction method was used to construct the models from the 3D modelling space. The specific steps were described as follow:

Step 1, a fundamental body of the modelling area was constructed by the map boundary polygon. The boundary polygon was extended to the modelling altitude and a series of contour lines were acquired. Through contour line connection and constrained Triangular Irregular Network (TIN) method, the side face and bottom face were constructed. With the terrain surface constructed by the Digital Elevation Models (DEM) points, the fundamental body was complete. boundaries. Then the implicit geological interfaces were constructed by substituting the boundaries' points and vectors into the HRBF surface.

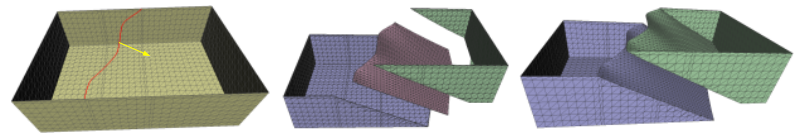

Figure 1. Fundamental body split by the geological interface

Step 3, the fundamental body was split into two fundamental bodies by the interface. During the split process, an interface boundary was acquired and the interface was reconstructed by vertical scanning line method (Figure 1) to make the interface pass through the boundaries.

Step 2, the attitudes were assigned to the corresponding boundaries and were transformed as the normal vectors of the

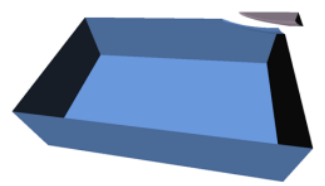

Forming:

Remaining:

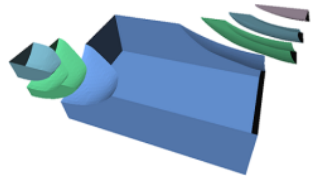

Forming:

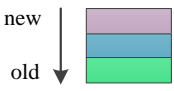

Remaining:

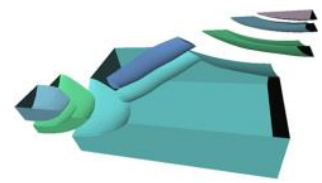

Forming:

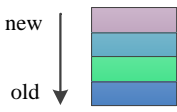

Remaining:

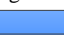

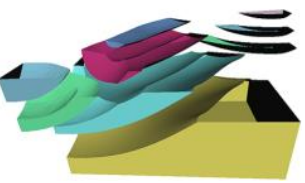

Forming:

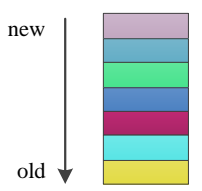

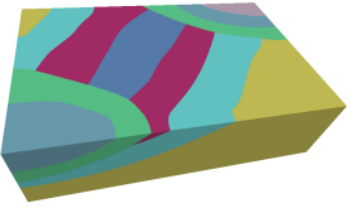

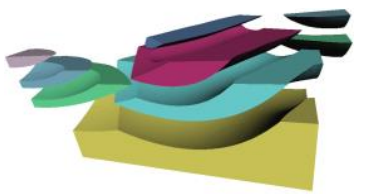

Figure 2. Sequential extraction of geological models from the fundamental body from new geologic time to old one.

Step 4 , by redoing the step 3 , every geological interface was used to split the corresponding fundamental body from new to old and all the geological bodies were constructed when all the interfaces participated in the extraction process (Figure 2).
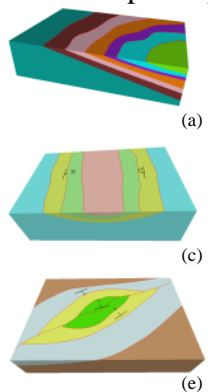
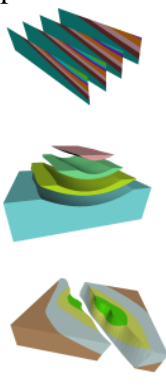
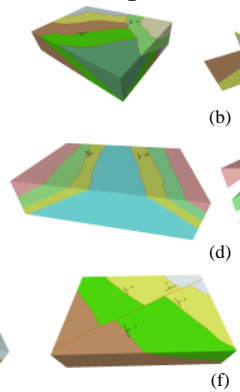
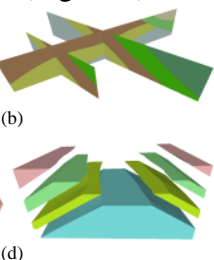

(b)
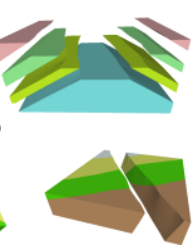

Figure 3. Simulation of typical geological structures; (a) conformable stratum model; (b) unconformable stratum model; (c) syncline model; (d) anticline model; (e) normal fault model; (f) parallel fault model.

\section{Modelling Experiments from Real Data and Other Applications}

Based on this workflow, a prototype system (Figure 3) was developed based on QT framework and OpenGL visualization technology to conduct these modelling experiments and a series of typical geological structures were simulated in our study, including conformable and unconformable stratum, folds, faults, etc. These models are shown in Figure 3, including the sections and explosive view of the models.

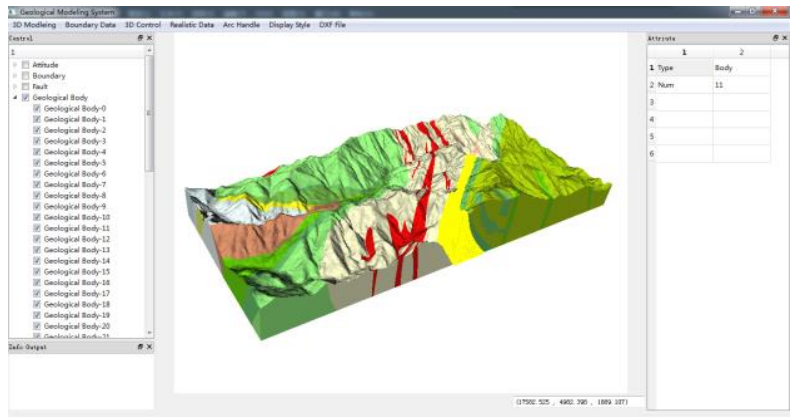

Figure 4. Geological modelling prototype system

\subsection{Examples of 3D Implicit Geological Modelling}

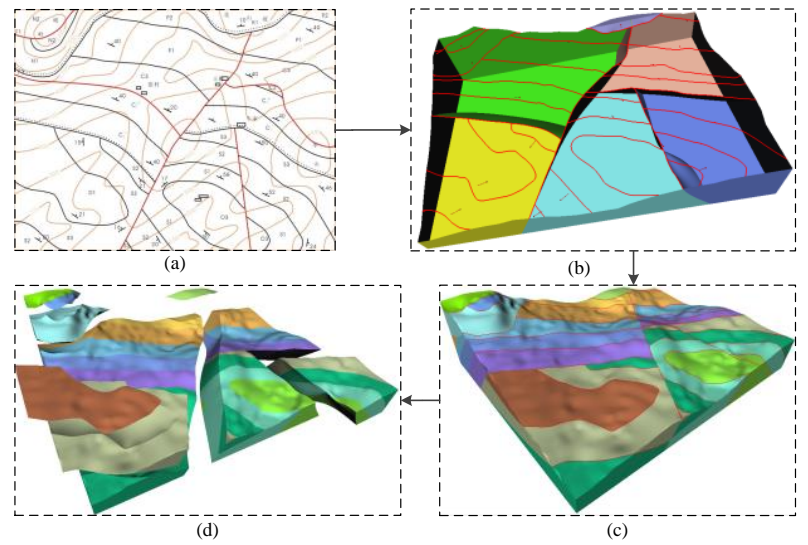

Figure 5. 3D regional geological modelling containing typical structures (folds, faults) from the 2D geological map; (a) 2D geological map; (b) geological framework modelling based on fault data; (c) integral regional geological model; (d) explosive view of geological units.

Some geological map was input into this prototype system, and 
the corresponding geological models were constructed through this workflow. Figure 5 shows the regional geological models of the Xinggang area in Chongqing, China. Typical folds and faults models were contained in these models. The geological map of a tunnel engineering area in Sichuan, China was also chosen in this research and the complex rock mass, stratum, dyke models were simulated in this experiment, shown in figure 6 .

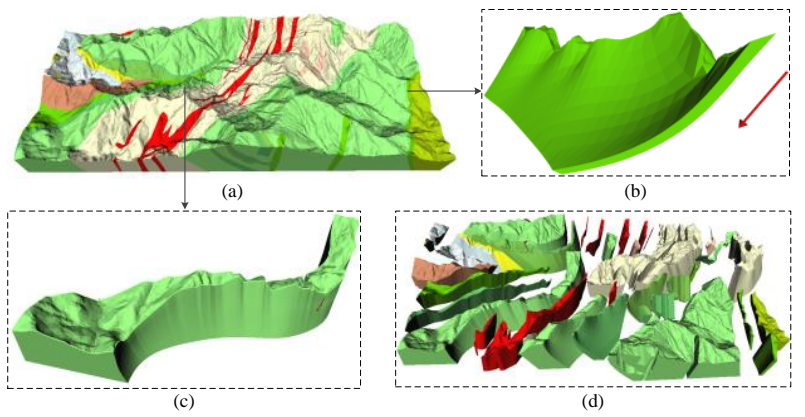

Figure 6. 3D regional geological modelling including rock mass, stratum, dykes etc. (a) regional geological model; (b) stratum model conformed to attitudes; (c) long-narrow rock mass model; (d) explosive view of geological units.

\subsection{D Implicit Mine Ore Body Modelling from Drill-hole}

Based on HRBF surface, ore bodies were constructed more flexibly and automatically from sampling mineral grade data collected by drill-holes. According to the exploitation cost of the ore, a boundary grade was given by modelers first. Based on the boundary grade, the sample sections whose grades were higher than boundary grade were chosen out. Then, the junction point of the adjacent sections whose grades distributed on both sides of the boundary grade was treated as the boundary point of the ore body. Meanwhile, to each boundary point, a unit vector point at the section with higher grade was regarded as the constrained vector of this boundary point. When the boundary points and normal vectors were substituted into the function, the implicit HRBF expression was calculated out. Based on this implicit function expression, the marching cube method was used to translate the implicit surface into triangle meshes and the models were visualized in the modelling system. Without any manual interactions, the models could be constructed and the stones inside the ore body were also constructed, which made the ore simulation and modelling updating very easily.

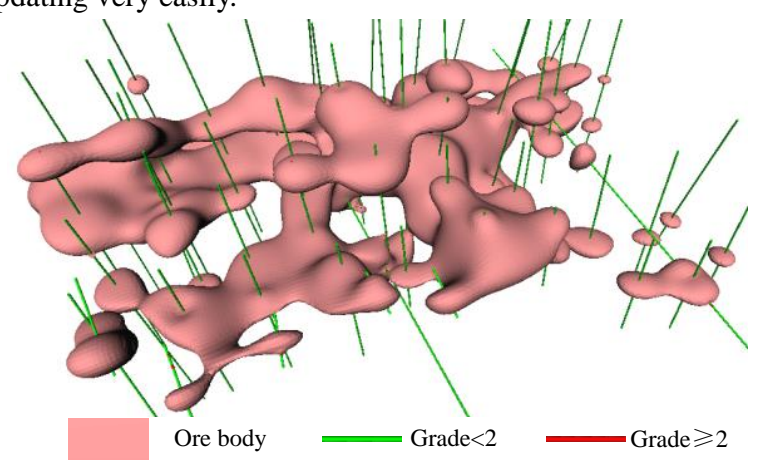

Figure 7. Ore body model constructed from drill-hole data

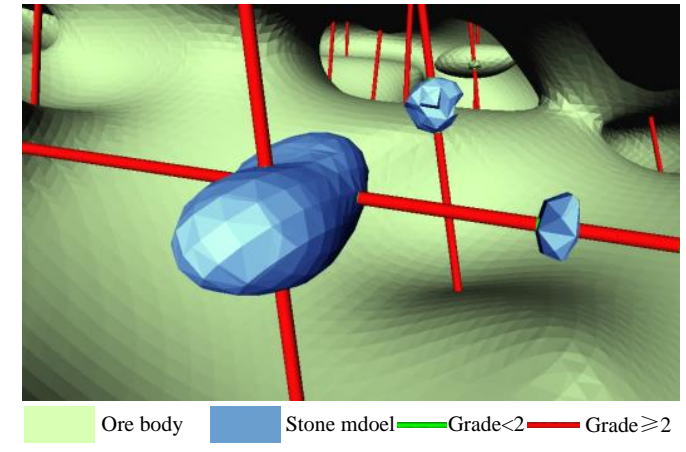

Figure 8. Stone model inside ore body

\subsection{D Implicit Geological Modelling from Geotechnical Engineering Drill-hole}

For the subsurface stratum modelling based on drill-hole data, an extraction method similar to the geological modelling workflow mentioned above was adopted in our study. First, the maximum and minimum coordinates of the modelling area were found out from the drill sample points and a bounding box model containing all the sample points was constructed. Then the lithology properties were assigned to each sample points and the corresponding sample points of each stratum interface were determined. To the points belonging to the same stratum interface, the normal vectors were calculated out (Pauly M, 2002), and the interface was constructed by substituting the sample points and vectors into the HRBF surface. Finally, each stratum model was extracted from the bounding box by the stratum interface model from new to old. The models constructed through this workflow were very smooth and models' edge was very regular. Furthermore, the pinch-out stratum were simulated very naturally and precisely.

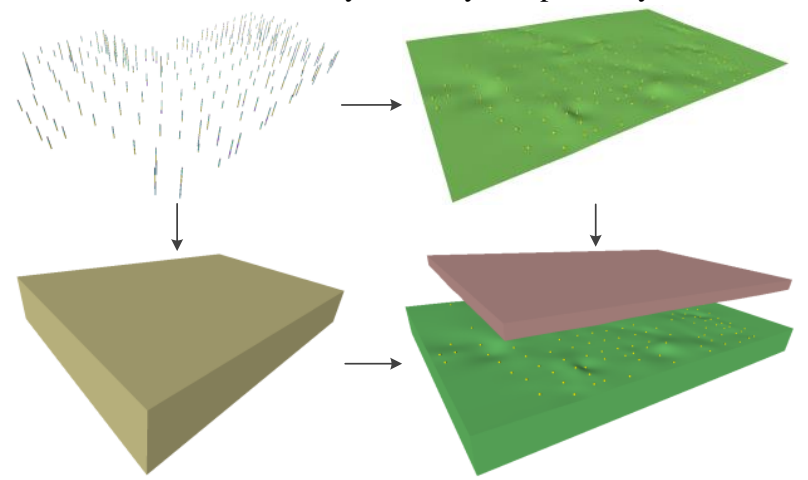

Figure 9. One stratum interface was constructed from the drill sample points and the bounding box model was split into two parts
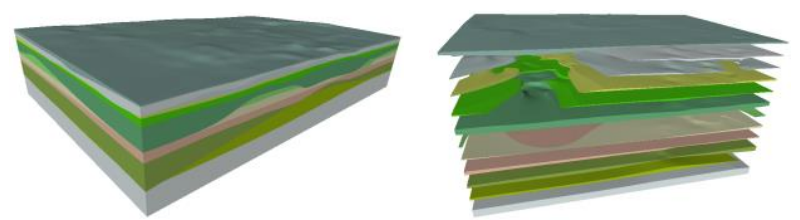

Figure 10. The stratum models were constructed after series of interface splitting from bounding box 


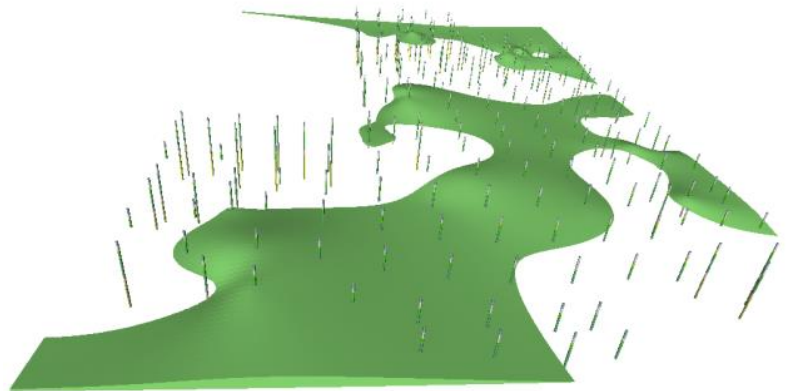

Figure 11. The pinch-out stratum model was simulated very naturally and precisely

\section{Conclusions and Discussion}

A very powerful spatial surface, Hermite radial basis function surface, was adopted to simulate the geological structures in our study. The extraction modelling workflow was taken in the 3D geological modelling to construct all the models while maintaining the topological relations between them. Next, ore body models and stratum models were constructed by HRBF surface from drill-hole data. Both 2D geological maps and drill-holes were honored by this interpolation method and the models were all constructed naturally and precisely.

Although HRBF surface performed well in simulating geological structures, some disadvantages were still unavoidable. Time-consuming computation for solving the HRBF equation was the bottle-neck of large data. Meanwhile, it was very difficult to translate the implicit surface into triangle meshes under geometric constraints.

\section{REFERENCES}

Alaei B. 2012. Seismic modeling of complex geological structures. INTECH Open Access Publisher, pp. 213-236.

Amorim, R.; Brazil, E.V.; Samavati, F.; Sousa, M.C. 2014. 3D geological modeling using sketches and annotations from geologic maps. In Proceedings of the 4th Joint Symposium on Computational Aesthetics, Non-Photorealistic Animation and Rendering, and Sketch-Based Interfaces and Modeling; ACM: New York, NY, USA, pp. 17-25.

Borghi A, Renard P, Courrioux G. 2015. Generation of 3D Spatially Variable Anisotropy for Groundwater Flow Simulations. Groundwater, pp. 1-4.

Calcagno P. 2015. 3D GeoModelling for a Democratic Geothermal Interpretation, in Proceedings World Geothermal Congress; Melbourne, Australia.

Cowan E J, Beatson R K, Ross H J, et al. 2003. Practical implicit geological modelling[C]//Fifth International Mining Geology Conference, pp. 17-19.

De Kemp E A, Sprague K B. 2003. Interpretive tools for 3-D structural geological modeling part I: Bezier-based curves, ribbons and grip frames[J]. GeoInformatica, 7(1), pp. 55-71.

Guo J, Wu L, Zhou W, et al. 2016. Towards Automatic and Topologically Consistent 3D Regional Geological Modeling from Boundaries and Attitudes[J]. ISPRS International Journal of Geo-Information, 5(2): 17.

Houlding S. 1994. 3D geoscience modeling: computer techniques for geological characterization[M]. London: Springer-Verlag.

Kazhdan M, Bolitho M, Hoppe H. 2006. Poisson surface reconstruction[C]//Proceedings of the fourth Eurographics symposium on Geometry processing. 7.

Kessler, H.; Mathers, S.; Sobisch, H.G. 2009. The capture and dissemination of integrated 3D geospatial knowledge at the British Geological Survey using GSI3D software and methodology. Comput. Geosci.35, pp. 1311-1321.

Macedo, I.; Gois, J.P.; Velho, L. 2011. Hermite radial basis functions implicits. Comput. Graphics Forum, 30, $27-42$.

McGaughey J, Milkereit B. 2007. Geological models, rock properties, and the $3 \mathrm{D}$ inversion of geophysical data. Proceedings of exploration.7, pp. 473-483.

Ming, J.; Pan, M.; Qu, H.; Ge, Z. 2010. GSIS: A 3D geological multi-body modeling system from netty cross-sections with topology. Comput. Geosci. 36, pp. 756-767.

Pauly M, Gross M, Kobbelt L P. 2002. Efficient simplification of point-sampled surfaces[C]//Proceedings of the conference on Visualization'02. IEEE Computer Society, pp. 163-170.

Qingyuan Li, ZHANG L, Zhan-ying W E I. 2013. On 3D geological modeling software development and discussions on several issues[J]. Journal Geology, 37(4), pp. 554-561.

Raiber M, White P A, Daughney C J, et al. 2012. Three-dimensional geological modelling and multivariate statistical analysis of water chemistry data to analyse and visualise aquifer structure and groundwater composition in the Wairau Plain, Marlborough District, New Zealand. Journal of Hydrology, 436, pp. 13-34.

Vance T C, Merati N, Mesick S M, et al. GeoModeler: Tightly linking spatially-explicit models and data with a GIS for analysis and geovisualization[C]//Proceedings of the 15th annual ACM international symposium on Advances in geographic information systems. ACM, 2007: 32 .

Wu L. 2004. Topological relations embodied in a generalized tri-prism (GTP) model for a 3D geoscience modeling system[J]. Computers \& Geosciences, 30(4), pp. 405-418.

Zanchi A, Francesca S, Stefano Z, et al. 3D reconstruction of complex geological bodies: Examples from the Alps[J]. Computers \& Geosciences, 2009, 35(1): pp. 49-69. 